\title{
LANGUAGE EVOLUTION: A BRIEF OVERVIEW
}

\author{
Dr Christine Cuskley \\ Centre for Behaviour and Evolution \& Linguistics Section \\ Newcastle University, Newcastle upon Tyne, UK \\ christine.cuskley@ncl.ac.uk
}

\begin{abstract}
This article provides a very brief overview of the study of language evolution, targeted at students early on in their studies in any field. The article begins by arguing that to understand language we must understand its evolution, and briefly shows how interest in the topic has expanded, particularly in the last 30 years. This leads to a closer look at what we now understand about language evolution, focusing on consensus and looking at major themes, particularly complimentary biological and cultural approaches. The article concludes with a look at the interacting genetic, cultural, and developmental timescales at play in the evolution of language.
\end{abstract}

The biologist Theodosius Dobzhansky famously said that "nothing in biology makes sense expect in the light of evolution" (Dobzhansky, 1973). Like the study of life, the study of human language quite arguably needs the light of evolution in order for us to truly understand it. Despite the evident importance of understanding language evolution, the serious study of the subject is in its relative infancy. Broadly, this is due to the seemingly intractable nature of the problem: spoken and signed languages predate written language by at least hundreds of thousands of years. These earlier forms leave few concrete fossils for us to study (Fitch, 2010).

Despite the difficulty of the problem, the study of language evolution has advanced considerably in recent years. This is due in large part to a resurgence of interest in the subject in the latter part of the 20th century on the heels of the cognitive revolution. For example, the figure below shows that over ten times as many articles were published on language evolution in just the first half of 2020 than 30 years earlier, in 1990.

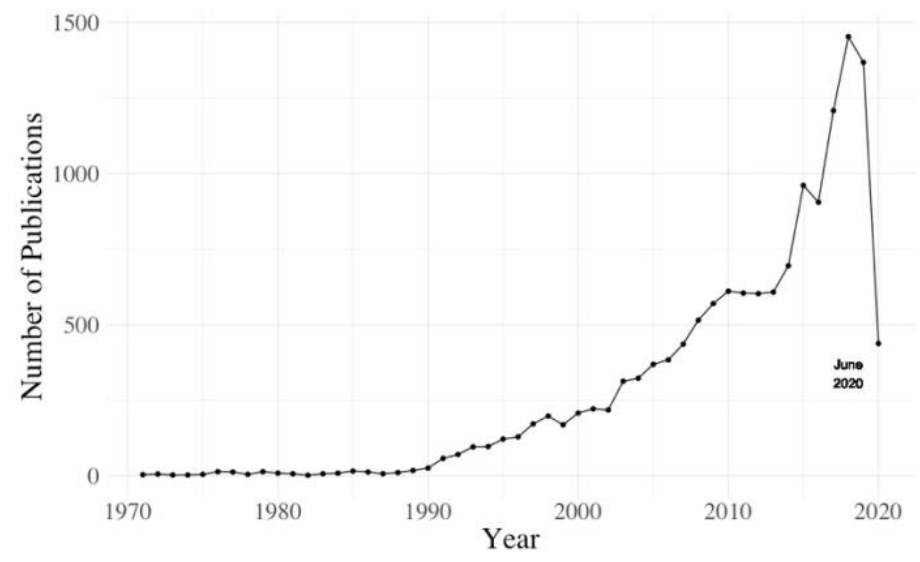

Figure 1 Number of publications over time with the topic 'language evolution' from 1970 to June 2020. Data retrieved from Web of Science, 11 June 2020. 
However, the question of language evolution remains largely open, with no fully complete theory holding sway over the field. This article will review major themes in the study of language evolution, touching upon methodological approaches which have aimed to overcome the intractability inherent in the problem.

\section{What makes language unique?}

Language is central to almost all human interaction, and is a uniquely human trait. A key component of understanding language evolution is to understand exactly what makes language unique. Very generally, this can be summed up in four major qualities: arbitrariness, generativity, displacement, and reflexiveness. These are just a handful of the design features originally identified by Hockett (1960)- but these are specifically the ones that are prime candidates for what sets human language apart from other animal communication stystems.

Language is arbitrary in that the signs are symbolic; word forms in language do not need to have any specific connection to their meanings. In other words, a rose, by any other name, would still smell as sweet. Among other known animal communication systems, this is relatively rare. More commonly, communicative signs are related to their meanings - for example, a deep growl usually means a large animal. Indeed, a growing body of research has suggested that while language is now largely arbitrary, it exhibits many kinds of non-arbitrariness (Dingemanse et al., 2015), and language may have gotten off the ground by utilizing forms that were motivated by the meanings they referenced (Cuskley \& Kirby, 2013).

Language is also generative, that is, language systems can be used to generate new sentences and words. The structure of language allows us to create theoretically infinite utterances (John knows that Mary knows that Sue knows... etc.), and word formation rules allow new forms to enter the language (e.g., podcast). Human language also allows for displacement: we can refer to things and events distant in space and time. Finally, language can be reflexive: it allows us to not only talk about ourselves, but about language itself. An open question is whether language is required for displacement and reflexiveness, or whether the ability to engage in displacement and reflexiveness supported the evolution of language. It is unlikely we will find a definitive answer to this question, but most researchers agree that whichever came first, there was likely a general ratchet effect between language and the cognitive abilities which supported or resulted from it.

\section{Major themes in language evolution}

The study of language evolution is inherently diverse and interdisciplinary, and has relied on input from psychology, linguistics, biology, archaeology, and complex systems science, among others. Although there is little consensus in a field of study as diverse and dynamic as language 
evolution, most researchers agree on two crucial points: (i) human language did, in fact, evolve, and (ii) it is unique among the other animal communication systems on earth. When exactly language evolved is not known, and some evidence shows that other hominid species (e.g., Neanderthals and Denisovans) likely also have had language, or at least something like it (Chen et al., 2019; Dediu \& Levinson, 2018). Currently, a broad consensus holds that language likely evolved sometime between 2 million years ago to 200,000 years ago - estimates vary.

Many researchers propose an intermediate stage prior to full-blown human language known as protolanguage (Smith, 2008; Tallerman, 2007). Protolanguage is generally characterised as having broad similarity to the language of young children and language trained apes: a confined vocabulary size, a focus on meanings related to concrete objects, actions, and attributes, and relatively free word order. Some theories have proposed that protolanguage was predominantly or entirely gestural (Corballis, 2017), but emerging consensus is that it was likely an integrated combination of speech and gesture, much like modern language (Kendon, 2017).

Conceptually, protolanguage prevents against the potential red herring of uniformitarianism: the broad assumption that language was the same when it evolved as it is now (Newmeyer, 2003). The push against this assumption dovetails with the broad consensus that language evolved at least somewhat gradually, having some continuity with other communication systems, rather than arising fully formed (Tallerman, 2014). An increasing minority of theorists propose a more discontinuous transition to language sometimes known as saltation - for example, some of Chomsky's early work on language evolution (Hauser et al., 2002) has been interpreted in this way (Jackendoff \& Pinker, 2005), though more recent clarifications line up more with a gradual evolutionary picture (Fitch, 2010).

This gradual view is supported by research in linguistics. Work in historical linguistics and language change indicates that human languages can change relatively rapidly - on the order of thousands if not hundreds of years (Greenhill et al., 2017). Therefore, language at its origin was likely both qualitatively and quantitatively different from its modern form. The study of modern language change and historical linguistics remains an important component of language evolution: understanding how languages change over time and the factors involved in language speciation can provide important insights in the study of language evolution more broadly.

Given these very broad swaths of agreement and disagreement, most research in language evolution focuses on the biological evolution of language or the cultural evolution of language. Biological perspectives focus mainly on evolution of the physical, neurological, or individual cognitive structures required for language. On the other hand, cultural perspectives focus more on language itself, and examine how social interactions between individuals may have shaped evolution of language. These different approaches are largely complimentary, painting an overall 
picture of language that, like many human behaviours, arises out of a complex interplay between nature and nurture.

\section{Biological Evolution of Language}

Biological accounts of the evolution of language emphasize the common properties across human languages to support a shared, genetic cognitive underpinning for language. Many accounts even argue that the genetic underpinning of language was specifically selected for, and gains support from the discovery of some specific genes related to language development. A relatively early example of a gene related to language comes from the human variant of the FoxP2 gene, which appears to have undergone strong selection in recent human evolution (Enard et al., 2002). FoxP2 governs oral and facial development, among other things, and mutated forms of the gene are directly related to an inherited condition known as selective language impairment (Fisher \& Scharff, 2009), which was discovered in part because it was prevalent in a family - known as the KE Family - that researchers began studying in the 1980s (Nudel \& Newbury, 2013). The fact that a language-related impairment seemed to be highly inherited led researchers to suspect some genetic underpinning that affected language directly and selectively, although the human genome wasn't fully mapped until the early 2000s (Green et al., 2015). Generally, genetic underpinnings of language are complex and this is an open area of research - what is known is that it is likely that the capacity for language is highly polygenetic, meaning it involves a complex interplay of many genes (for further reading, see Dediu \& Christiansen, 2016).

Some biological accounts refer to a biological language faculty as the primary phenomenon that must be studied or explained in order to understand language evolution. In this view, the core of the language faculty is a set of computational abilities that relate to recursion, a property of human languages which allows us to create and understand theoretically infinite utterances (Hauser et al., 2002). Other biological accounts focus more concretely on specific physiological adaptations such as the organization of the human vocal tract (de Boer, 2005). Our vocal tract differs markedly from that of our closest animal relatives, chimpanzees, for example, allowing us to articulate a broader set of sounds than chimpanzees can, and suggesting that a reorganization of the vocal tract must have conveyed an adaptive advantage (Lieberman, 2007). Recent research even suggests that human oral physiology was shaped by changed to the human diet as agriculture became more widespread, leading in turn to subtle changes in the sounds prevalent in language (Blasi et al., 2019).

Theories of the evolution of language often focus on evidence from non-human animals, looking at communication systems elsewhere in the animal kingdom in order to better understand 
our own. Comparing human language to the communicative behavior of other animals focuses on both analogous structures (those similar to other animals, but which likely developed independently under different selective pressures) and homologous structures (those shared with our closest animal relatives via shared ancestry).

Analogous structures have the potential to reveal what selective pressures led us to develop language. For example, complex syntactically structured birdsong emerged primarily as a result of sexual selection (Berwick et al., 2011), leading some researchers to suggest sexual selection also played a role in language evolution (Zawidzki, 2006). Other evidence from birdsong learning shows that vocal learning is motivated and reinforced via specific oxytocin pathways, potentially shedding light on how social vocal learning may work in humans (Theofanopoulou et al., 2017). Other animals also have surprising parallels to certain aspects of human language. Bees, for example, engage in communicative "dance" inside the hive to convey information to other bees regarding the direction, distance, and attractiveness of pollen sources, showing an impressive level of displacement (Root-Bernstein, 2010). Dolphins have a complex communication system researchers have yet to decipher completely - research is ongoing. However, dolphins are known to have "name calls' - clicks and whistles they use to refer to specific individuals - indicating some level of reflexiveness (Janik \& Sayigh, 2013).

The study of homologies of language, via our primate relatives, has been considerably more in depth. The communicative and cognitive behavior of our closest relatives in the animal kingdom reveal what aspects of language may have been present in our last common ancestor (e.g., our last common ancestor with chimpanzees lived approximately 6-7 million years ago). This gives us a more specific idea of what about human language is unique, allowing a more focused study of exactly what evolved to give us language. A homologous approach focuses mainly on studying the communicative behavior of other great apes (e.g., chimpanzees and gorillas), but also some other primate species (e.g., alarm calls in monkeys).

Studies of behavior in the wild have revealed that our closest relatives - chimpanzees - as well as other great apes (gorillas and orangutans) have a far richer natural communicative system than previously thought, including a diverse set of calls, facial signaling, and gestures (Byrne et al., 2017). These communication systems contribute to complex social group dynamics and coordinated behavior (e.g., wild chimpanzees' cooperative hunting of red colobus monkeys, Newton-Fisher et al., 2002). Even the alarm calls of our more distant monkey relatives are sophisticated, allowing for intentional deception. For example, a low-ranking tufted capuchin may send out a false alarm call to distract others in the troop from a high-quality food source, thereby keeping the food to itself (Wheeler, 2009). 
Studies of captive primates have also illuminated issues surrounding language evolution, most famously in the case of Kanzi the bonobo chimpanzee. Kanzi has learned to use hundreds of lexigrams (arbitrary graphics representing meanings) expressively, can understand thousands of spoken words, and even shows some sensitivity to word order (Kako, 1999). However, other chimpanzee studies have been less successful, most notably a chimpanzee named Nim Chimpsky (a clever play on the famous Noam Chomsky) acquired a fairly impressive sign language vocabulary, but never showed definitive sensitivity to syntax (Hess, 2008).

Although these studies have revealed sophisticated communicative behavior in our ape and monkey relatives, they also reveal specific shortcomings. Ape communication systems in the wild are more complex and rich than previously thought, but they show little evidence of generativity, displacement, or reflexiveness. Although the use of alarm calls for deception has been documented, such innovative use is not widespread, and alarm call systems are still limited to only a handful of "meanings". Based on the behavior of language trained apes such as Kanzi, it would appear that some primates have the capacity for many of the unique aspects of language, but it is, at the very least, quantitatively limited. Kanzi's overall linguistic repertoire seems to have plateaued, and he does not show the same understanding of hierarchical structure as a human toddler (18-24 months old), who nonetheless has not yet completely acquired fluency in language (Truswell, 2017). Moreover, data from other language-trained chimpanzees is less encouraging, indicating that there may be considerable individual variation in terms of language capacity among non-human primates. Regardless, for language-like behavior to emerge, chimpanzees require unusually rich and humanlike developmental conditions. Kanzi may be a particularly cognitively advanced chimpanzee - it is difficult to know if this is because of his language training, or if he was successful in language training precisely because he is particularly intelligent.

All of this points to some special biological adaptation in humans which allows us to develop language. While our closest relatives, chimpanzees, are able to engage in many of the unique aspects of language under particularly rich developmental conditions, they appear unable to do so to the same degree as even very young humans who are just beginning to learn language. Analogous structures highlight that no single aspect of linguistic ability exists as an island; rather, a suite of abilities is necessary for the full richness of human language. Bee dance allows for displacement, primate communications systems have limited meaning, bird song exhibits complex hierarchical structure, and there is some evidence of reflexiveness in dolphins — but only human language seems to combine all of these into a single, uniquely powerful communication system. Overall, biological approaches to the evolution of language point to a perfect storm of social and cognitive abilities which support human language. 


\section{Cultural Evolution of Language}

Biological accounts of language evolution provide essential context of the human language faculty within the rest of the animal kingdom, but they focus primarily on aspects of language that operate at the individual level. Cultural approaches to language origins emphasize the role of interaction and learning in the evolution of language, highlighting similarities and differences across languages to identify cultural pressures which constrain the evolution of language in populations. Cultural accounts consider language behavior and structure to be the phenomena which should be the object of investigation in language evolution, and tend not to focus on a specific language faculty. Methodologically, they rely primarily on computational models, psychological experiments, and linguistic data, but also draw to some extent on developmental and comparative data.

Computational models in language evolution generally focus on the hypothesis that cultural interaction and learning biases played a large role in the evolution of language. As an illustrative metaphor, researchers often refer to the notion that the language evolves and adapts to the selective pressures imposed by learners (Christiansen \& Chater, 2008). In order to "survive," a language must be learnable, or else it will not be passed on to successive generations of speakers or signers. A successful language must also be useful: it must express the many meanings its users need to communicate. These two pressures combine to favour languages which are structured, since structure allows for maximum learnability and expressivity (Kirby et al., 2015). For example, learning a single rule (e.g., add $-s$ to to the singular to form the plural) is less cognitively demanding that learning a rote list (i.e., if all plurals were irregular, such as goose-geese, ox-oxen). Simultaneously, this rule generalizes to new forms (e.g., the plural of selfie is uncontroversially selfies), allowing for increased expressive power. Much of the evidence for these accounts comes from agent-based modeling and more traditional psychological experiments, particularly in artificial language learning.

A pioneering model examining the role of interaction in language evolution is known as the Naming Game (see Baronchelli, 2016 for an overview). The Naming Game focuses on a population of interacting artificial agents tasked with labelling a particular meaning - agents begin by generating random strings for the meaning. Although communicative success within the population is initially low (that is, agents do not agree on which label the meaning should have), a simple bias to discard all previous labels when they meet an agent which shares one of their labels leads to high communicative success over time. In other words, this simple bias allows agents to converge on a shared conventional label for a meaning. This finding emphasizes the role communicative function in language evolution: the pressure to communicate successfully, and the process of communication itself, can shape language over time. 
Another social learning perspective focuses on coordination in learning instead of interaction. Language learners do not receive disembodied input, rather, they learn from the output of other language users. The iterated learning model begins with agents learning a subset of an initially random artificial vocabulary (Kirby \& Hurford, 2002). A subset of the output of this first "generation" of agents then becomes the input of the next generation. Since agents receive only a subset of the input - but are tested the entire vocabulary - they generalize patterns to unseen items (much like knowing blog-blogs leads to selfie-selfies) and structure begins to accumulate within the vocabulary over successive generations of learners.

The iterated learning model has also been implemented experimentally: participants were tasked with learning initially random pairings of nonsense words and meanings, and their output was transmitted as the learning input for the next participant (Kirby et al., 2008). As in the computational implementation, results showed that initially random languages become structured (i.e., words which shared aspects of forms referred to similar meanings), supporting the notion that weak individual biases are amplified by transmission and shape the structure of language. This line of research highlights the key role of cultural transmission in language evolution. Each individual agent or participant only has a small bias towards structure, but this bias is amplified by transmission, creating a highly structured language. These approaches emphasize the key role that cultural processes can play in creating linguistic meaning and structure.

This kind of approach has also been extended to primates. Instead of a vocabulary, this study tasked baboons with learning simple random patterns on a grid with 16 cells (Claidière et al., 2014). Like in the model and experiment outlined above, the baboons then reproduce the simple patterns in a testing phase. Their test output then becomes the input for another baboon, creating a 'chain' of learning which includes several individuals. At the end of these chains, the patterns are no longer random, but exhibit structure - specifically, they resemble 'tetronimoes' - the shapes from the classic game Tetris, which involve 4 consecutive filled cells (see also, Saldana et al., 2019). Broadly, this shows that while these kinds of cultural evolution processes may have been instrumental in language evolution, they likely extend well beyond that. Cultural evolution is generally understood to be important for a variety of animals, indicating fairly deep evolutionary roots (Creanza et al., 2017).

\section{Interacting Timescales}

Although biological and cultural perspectives are often characterized as alternatives, the emerging view in language evolution is that biological and cultural factors interact with individual learners to shape language. From the biological perspective, there is broad agreement that at the very least some biological adaptation primes us for language, since no other species that we know 
of has all of the key components of language in a single system. On the other hand, language requires some cultural input in development, as evidenced by unfortunate cases of language deprived children who have persistent difficultly with structure in language in particular (Vyshedskiy et al., 2017).

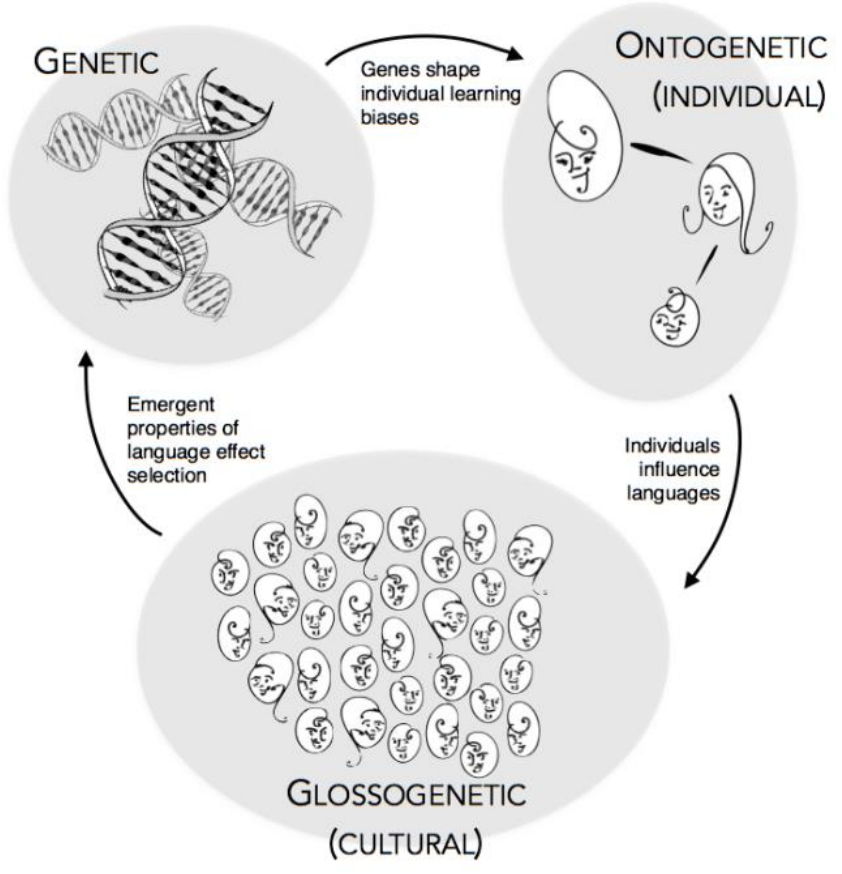

Figure 2 Interacting timescales of language evolution. Adapted with permission from Kirby (2002).

Current theory in language evolution has largely shifted towards characterizing the interaction of biological and cultural factors (Figure 1). Any biological underpinnings of language necessarily evolve on the relatively slow genetic timescale, on the order of thousands of years at a minimum. The cultural or glossogenetic timescale on the other hand, changes rapidly; for example, new words emerge and others die within mere years. The individual or ontogenetic timescale represents language development - while human children are undoubtedly primed to learn language biologically, their linguistic input comes from the cultural realm, and their output in turn feeds back into the cultural timescale. The cumulative language behavior of a population of individuals has the potential to affect the input of new learners, and thus shape selective pressures at work on the genetic scale, resulting in complex and ongoing co-evolution of genes and culture (Richerson, 2017).

\section{Conclusion}

The study of language evolution is not only essential for understanding language, but essential to understanding what it means to be human. It is a growing and vibrant field with vital contributions from a variety of disciplines and methodologies. The extent to which biological and 
cultural factors contributed to the evolution of language is a subject of active debate in the field, but there is broad consensus that an interaction between individuals, culture, and biology hold the key to a better understanding of how language evolved. This understanding requires a joint effort across a variety of disciplines, which has grown in particular over the last thirty years and continues to expand today.

\section{References}

Baronchelli, A. (2016). A gentle introduction to the minimal Naming Game. Belgian Journal of Linguistics, 30(1), 171192. https://doi.org/10.1075/bj1.30.08bar

Berwick, R. C., Okanoya, K., Beckers, G. J. L., \& Bolhuis, J. J. (2011). Songs to syntax: The linguistics of birdsong. Trends in Cognitive Sciences, 15(3), 113-121. https://doi.org/10.1016/j.tics.2011.01.002

Blasi, D. E., Moran, S., Moisik, S. R., Widmer, P., Dediu, D., \& Bickel, B. (2019). Human sound systems are shaped by post-Neolithic changes in bite configuration. Science, 363(6432). https://doi.org/10.1126/science.aav3218

Byrne, R. W., Cartmill, E., Genty, E., Graham, K. E., Hobaiter, C., \& Tanner, J. (2017). Great ape gestures: Intentional communication with a rich set of innate signals. Animal Cognition, 20(4), 755-769. https://doi.org/10.1007/s10071-017-1096-4

Chen, F., Welker, F., Shen, C.-C., Bailey, S. E., Bergmann, I., Davis, S., Xia, H., Wang, H., Fischer, R., Freidline, S. E., Yu, T.-L., Skinner, M. M., Stelzer, S., Dong, G., Fu, Q., Dong, G., Wang, J., Zhang, D., \& Hublin, J.-J. (2019). A late Middle Pleistocene Denisovan mandible from the Tibetan Plateau. Nature, 569(7756), 409-412. https://doi.org/10.1038/s41586-019-1139-x

Christiansen, M. H., \& Chater, N. (2008). Language as shaped by the brain. Behavioral and Brain Sciences, 31(5), 489509. https://doi.org/10.1017/S0140525X08004998

Claidière, N., Smith, K., Kirby, S., \& Fagot, J. (2014). Cultural evolution of systematically structured behaviour in a non-human primate. Proceedings of the Royal Society B: Biological Sciences, 281(1797), 20141541. https://doi.org/10.1098/rspb.2014.1541

Corballis, M. C. (2017). A Word in the Hand: The Gestural Origins of Language. In M. Mody (Ed.), Neural Mechanisms of Language (pp. 199-218). Springer US. https://doi.org/10.1007/978-1-4939-7325-5_10

Creanza, N., Kolodny, O., \& Feldman, M. W. (2017). Cultural evolutionary theory: How culture evolves and why it matters. Proceedings of the National Academy of Sciences, 114(30), 7782-7789. https://doi.org/10.1073/pnas.1620732114

Cuskley, C., \& Kirby, S. (2013). Synesthesia, cross-modality, and language evolution. In The Oxford handbook of synesthesia (pp. 869-899). Oxford University Press. 
de Boer, B. (2005). Evolution of Speech and Its Acquisition. Adaptive Behavior, 13(4), 281-292. https://doi.org/10.1177/105971230501300405

Dediu, D., \& Christiansen, M. H. (2016). Language Evolution: Constraints and Opportunities From Modern Genetics. Topics in Cognitive Science, 8(2), 361-370. https://doi.org/10.1111/tops.12195

Dediu, D., \& Levinson, S. C. (2018). Neanderthal language revisited: Not only us. Current Opinion in Behavioral Sciences, 21, 49-55. https://doi.org/10.1016/j.cobeha.2018.01.001

Dingemanse, M., Blasi, D. E., Lupyan, G., Christiansen, M. H., \& Monaghan, P. (2015). Arbitrariness, Iconicity, and Systematicity in Language. Trends in Cognitive Sciences, 19(10), 603-615. https://doi.org/10.1016/j.tics.2015.07.013

Dobzhansky, T. (1973). Nothing in Biology Makes Sense except in the Light of Evolution. The American Biology Teacher, 35(3), 125-129. JSTOR. https://doi.org/10.2307/4444260

Enard, W., Przeworski, M., Fisher, S. E., Lai, C. S. L., Wiebe, V., Kitano, T., Monaco, A. P., \& Pääbo, S. (2002). Molecular evolution of FOXP2, a gene involved in speech and language. Nature, 418(6900), 869-872. https://doi.org/10.1038/nature01025

Fisher, S. E., \& Scharff, C. (2009). FOXP2 as a molecular window into speech and language. Trends in Genetics, 25(4), 166-177. https://doi.org/10.1016/j.tig.2009.03.002

Fitch, T. (2010). The Evolution of Language. Cambridge University Press.

Green, E. D., Watson, J. D., \& Collins, F. S. (2015). Human Genome Project: Twenty-five years of big biology. Nature News, 526(7571), 29. https://doi.org/10.1038/526029a

Greenhill, S. J., Wu, C.-H., Hua, X., Dunn, M., Levinson, S. C., \& Gray, R. D. (2017). Evolutionary dynamics of language systems. Proceedings of the National Academy of Sciences, 114(42), E8822-E8829. https://doi.org/10.1073/pnas.1700388114

Hauser, M. D., Chomsky, N., \& Fitch, W. T. (2002). The Faculty of Language: What Is It, Who Has It, and How Did It Evolve? Science, 298(5598), 1569-1579. https://doi.org/10.1126/science.298.5598.1569

Hess, E. (2008). Nim Chimpsky: The Chimp Who Would Be Human. Random House Publishing Group.

Hockett, C. F. (1960). The Origin of Speech. Scientific American, 203(3), 88-97. JSTOR. https://doi.org/10.2307/24940617

Jackendoff, R., \& Pinker, S. (2005). The nature of the language faculty and its implications for evolution of language (Reply to Fitch, Hauser, and Chomsky). Cognition, 97(2), 211-225. https://doi.org/10.1016/j.cognition.2005.04.006

Janik, V. M., \& Sayigh, L. S. (2013). Communication in bottlenose dolphins: 50 years of signature whistle research. Journal of Comparative Physiology A, 199(6), 479-489. https://doi.org/10.1007/s00359-013-0817-7 
Kako, E. (1999). Elements of syntax in the systems of three language-trained animals. Animal Learning \& Behavior, 27(1), 1-14. https://doi.org/10.3758/BF03199424

Kendon, A. (2017). Reflections on the "gesture-first" hypothesis of language origins. Psychonomic Bulletin \& Review, 24(1), 163-170. https://doi.org/10.3758/s13423-016-1117-3

Kirby, S. (2002). Natural language from artificial life. Artificial Life, 8(2), 185-215. https://doi.org/10.1162/106454602320184248

Kirby, S., Cornish, H., \& Smith, K. (2008). Cumulative cultural evolution in the laboratory: An experimental approach to the origins of structure in human language. Proceedings of the National Academy of Sciences, 105(31), 10681-10686. https://doi.org/10.1073/pnas.0707835105

Kirby, S., \& Hurford, J. R. (2002). The Emergence of Linguistic Structure: An Overview of the Iterated Learning Model. In A. Cangelosi \& D. Parisi (Eds.), Simulating the Evolution of Language (pp. 121-147). Springer. https://doi.org/10.1007/978-1-4471-0663-0_6

Kirby, S., Tamariz, M., Cornish, H., \& Smith, K. (2015). Compression and communication in the cultural evolution of linguistic structure. Cognition, 141, 87-102. https://doi.org/10.1016/j.cognition.2015.03.016

Lieberman, P. (2007). The Evolution of Human Speech: Its Anatomical and Neural Bases. Current Anthropology, 48(1), 39-66. https://doi.org/10.1086/509092

Newmeyer, F. (2003). What can the field of linguistics tell us about the origins of language? In S. Kirby \& M. H. Christiansen (Eds.), Language Evolution (pp. 58-76). Oxford University Press.

Newton-Fisher, N. E., Notman, H., \& Reynolds, V. (2002). Hunting of Mammalian Prey by Budongo Forest Chimpanzees. Folia Primatologica, 73(5), 281-283. https://doi.org/10.1159/000067454

Nudel, R., \& Newbury, D. F. (2013). Foxp2. WIREs Cognitive Science, 4(5), 547-560. https://doi.org/10.1002/wcs.1247

Richerson, P. J. (2017). Cultural Evolution and Gene-Culture Coevolution. Evolutionary Studies in Imaginative Culture, 1(1), 89-92. JSTOR. https://doi.org/10.26613/esic.1.1.17

Root-Bernstein, M. (2010). Displacement activities during the honeybee transition from waggle dance to foraging. Animal Behaviour, 79(4), 935-938. https://doi.org/10.1016/j.anbehav.2010.01.010

Saldana, C., Fagot, J., Kirby, S., Smith, K., \& Claidière, N. (2019). High-fidelity copying is not necessarily the key to cumulative cultural evolution: A study in monkeys and children. Proceedings of the Royal Society B: Biological Sciences, 286(1904), 20190729. https://doi.org/10.1098/rspb.2019.0729

Smith, A. D. M. (2008). Protolanguage reconstructed. Interaction Studies, 9(1), 100-116. https://doi.org/10.1075/is.9.1.08smi 
Tallerman, M. (2007). Did our ancestors speak a holistic protolanguage? Lingua, 117(3), 579-604. https://doi.org/10.1016/j.lingua.2005.05.004

Tallerman, M. (2014). No syntax saltation in language evolution. Language Sciences, 46, 207-219. https://doi.org/10.1016/j.langsci.2014.08.002

Theofanopoulou, C., Boeckx, C., \& Jarvis, E. D. (2017). A hypothesis on a role of oxytocin in the social mechanisms of speech and vocal learning. Proceedings of the Royal Society B: Biological Sciences, 284(1861), 20170988. https://doi.org/10.1098/rspb.2017.0988

Truswell, R. (2017). Dendrophobia in Bonobo Comprehension of Spoken English. Mind \& Language, 32(4), $395-415$. https://doi.org/10.1111/mila.12150

Vyshedskiy, A., Mahapatra, S., \& Dunn, R. (2017). Linguistically deprived children: Meta-analysis of published research underlines the importance of early syntactic language use for normal brain development. Research Ideas and Outcomes, 3, e20696. https://doi.org/10.3897/rio.3.e20696

Wheeler, B. C. (2009). Monkeys crying wolf? Tufted capuchin monkeys use anti-predator calls to usurp resources from conspecifics. Proceedings of the Royal Society B: Biological Sciences, 276(1669), 3013-3018. https://doi.org/10.1098/rspb.2009.0544

Zawidzki, T. W. (2006). Sexual selection for syntax and kin selection for semantics: Problems and prospects. Biology and Philosophy, 21(4), 453-470. https://doi.org/10.1007/s10539-005-9000-z 